\title{
Computer Vision Systems for Content-based Image Classification
}

\author{
Malay Bhatt ${ }^{1, *}$, Tejas Patalia $^{2}$ \\ ${ }^{1}$ Department of Computer Engineering, Dharmsinh Desai University, Nadiad, India \\ ${ }^{2}$ Department of Computer Engineering, V.V.P. Engineering College, Rajkot, India
}

\begin{abstract}
In this paper, computer vision based Content-Based Image Classification systems have been described which are useful in various service and product industries. We have proposed Confidence Co-occurrence Matrix, which is a modification of Generalized Co-occurrence Matrix. The proposed framework merges properties of Confidence Cooccurrence Matrix along with other features such as RGB and HSV Histograms, Local Binary Pattern and Canny's edge detection approach. Proposed approach creates a fixed- size descriptor of size 1632. Once a feature vector has been constructed, classification is performed using Linear Support Vector Machine. The System is tested on four different wellknown datasets namely, sport events Database, Flavia Leaf Dataset, Leeds Butterfly Dataset and Birds Dataset . The proposed system is implemented in MATLAB and achieves an average class accuracy of $96 \%, 99 \%, 95 \%$ and $95 \%$ for the four datasets respectively .
\end{abstract}

Keywords Classification, Edge Detection, Confidence Co-Occurrence Matrix, Histogram, Local Binary Pattern, Support Vector Machine.

AMS 2010 subject classifications 68U10, 93E10.

DOI: $10.19139 /$ soic-2310-5070-669

\section{Introduction}

In the last decade, Content-based Image classification has attracted people from both academia and industry. The task is to automatically classify an image by feature extraction and assignment of a label. The Content-based Image Classification produces many successful applications in areas like Agriculture, Music, Bio-metric, and Medical science to name the few.

Any activity done under specific environment, with the help of some objects, is widely known as 'event'. The activity can be carried out by humans (singing, cooking etc.) or by the nature (earthquake, flood etc.) or by any human-made objects (automated task or task carried out by robots). There are three types of dataset available for event classification: video, audio, and image. As many people capture events by clicking pictures, it is not always necessary to have the video information available. The event recognition from images is more challenging task as compared to background scene classification and object recognition. The remainder of this paper is organized as: Section 2 covers literature review, Section 3 gives a detailed view of the proposed system and explains Confidence Co-occurrence Matrix in detail, Section 4,5,6 and 7 discuss datasets and experimental setup, Section 8 describes results and comparisons among chosen classifiers and Section 9 concludes our work

\footnotetext{
*Correspondence to: Malay Bhatt (Email: malaybhatt202@yahoo.com)
}

ISSN 2310-5070 (online) ISSN 2311-004X (print)

Copyright (C) 2019 International Academic Press 


\section{Literature Survey}

Indoor and outdoor scene classification experiments were described in [5]. In the beginning, classification methods were using global spatial features such as color and texture histograms[13]. After that approach, the bag-ofvisual words (BoVW) model became very popular. The BoVW, which treats an image as a document, is an unordered collection of local features. The limitation of the BoVW is that it does not incorporate spatial layout of features. Based on approximate global geometric correspondence, Spatial Pyramid Matching (SPM) framework was designed. The SPM approach partitions an image into increasingly fine sub-regions and computes histogram of local features in each sub-region. Based on this SPM framework, may extensions have been proposed [14]. In [6], a global feature known as completed Local Binary Pattern along with multi-scale resolution was adopted for land-scene classification.

High-resolution satellite scene classification based on multiple feature combination was carried out in [7]. They used two-stage linear support vector machine. In the first stage, they combined sparse coding, Local Ternary Pattern histogram fourier, color histogram, and Scale Invariant Feature Transform features and generated probability images with the help of SVM. They tested proposed approach on 19-class satellite scene dataset. Each class has 50 images, with sizes of $600 \times 600$ pixels. They considered 30 images for training per class. The proposed method gave very promising performance.

Li, L.J. and Fei-Fei, L. first attempted to classify events in static images by integrating scene and object recognition. An event is defined in a static image as an activity performed by the humans under specific environment. They considered three control models: Scene only, Object only, and Object+Geometry models. The full model which was an integration of the three models, gave $73.4 \%$ classification accuracy for whole event class. They took randomly selected 70 samples from each class for the training and randomly selected 60 samples from each class for the testing. They created database of 8 widely varied sport events [1].

Hierarchical Matching Pursuit (HMP) leans meaningful multi-level representations from images layer by layer. HMP builds a feature hierarchy which contains three modules: batch tree orthogonal matching pursuit, spatial pyramid matching, and contrast normalization. HMP is also scalable and can handle full size images also. HMP outperforms SIFT based generative graphic model [1], SIFT based single layer sparse coding and also object bank [2] approach significantly. The accuracy achieved by HMP is $85.7 \%$ with 70 images in the training and 60 images in the testing for UIUC Sports dataset [4].

In remote sensing image analysis, high-resolution satellite images and hyperspectral images play vital role. The content-based high resolution satellite image classification is described in [15]. Machine learning models based on the deep learning approach seems promising in recent time. A novel Condition Random Field (CRF) model with the potentials defined over the deep features produced by a Deep Belief Network is proposed in [21]. In this paper, contextual information is clubbed with deep representation and novel DBN-CRF model has been proposed to improve classification accuracy using hyperspectral images. They achieved $92.15 \%$ overall accuracy over Indian Pines Dataset and $94.02 \%$ overall accuracy over University of Pavia Dataset.

Bhatt M.S. and Patalia T. P. used Generalized Co-occurrence Matrix (GCM), obtained from HSV color space, having 64 gray-levels with various distance values (3,6,9,12 and 15) as input to Genetic Programming System. Genetic Programming evolved spatial descriptor using 15 Generalized Co-Occurrence matrices as terminals have been implemented with 7 functions and 2 operators. Each GCMs having size of $64 \times 64$ is considered as input. Obtained Genetic programming evolved spatial descriptor is tested on manually created Indian monuments database having four classes, namely 'Taj Mahal', 'Qutub Minar', 'Golden Temple' and 'India Gate'. Fitness function used in GP system is linear SVM with 10 fold cross validation. They obtained accuracy of 92\%[17]. They have also extended the work of Indian monuments classification and achieved 97\% classification accuracy [16].

Dubey et al. [18] performed species and variety classification of fruits and vegetables using Improved Summation and Difference Histogram (ISDH) technique. They performed k-means approach, with value '2' for the variable ' $k$ ', to segment the image into the foreground and background. The dataset contains fifteen different classes with a total of 2633 images. Forty images from each class are used to train the Multi-Class Support Vector Machine while the remaining images from each class are used to test the developed system. In the Multi-Class Support Vector 
Machine, accuracy is $95 \%$ for the 'Fuji Apple', for 'Nectarine' accuracy is $97 \%$, for 'Spanish Pear' accuracy is $96 \%$, and for the remaining classes, accuracy is about $98 \%$.

Dubey et al. [19] performed disease classification of fruits using Improved Summation and Difference Histogram (ISDH) technique. They performed k-means approach, with value '4' for the variable ' $k$ ', to segment defected area. Blotched, Rotten, Scabbed and Normal are different types of disease present in the apple. There is a total of 391 images. Sixty images from each class are used to train the multiclass Support Vector Machine while the remaining images from each class are used to test the SVM. They compared the ISDH method with the state of the art techniques like Global Colour Histogram, Colour Coherence vector, and Unser's descriptor. The developed system produced $92 \%$ average accuracy in an RGB colour space and 97\% in an HSV colour space. They reported an accuracy of $99 \%$ when Gradient features were combined with ISDH features. The authors used $67 \%$ of the total images from the database in the training process.

Dubey et al. [20] performed species and variety classification of fruits and vegetables using the Global Colour Histogram, Colour Coherence Vector, Colour Difference Histogram, Structure Element Histogram, Local Binary Pattern, Local Ternary Pattern, and Complete Binary Pattern Techniques. They performed k-means approach with value ' 2 ' for the variable ' $k$ ' to segment the image in the foreground and background. The dataset consists of fifteen different classes with a total of 2633 images. Sixty images from each class are used in the training while the remaining are used in the testing using the Multi-Class Support Vector Machine. The authors achieved 93\% accuracy.

Since each type of features has its own pros and cons, we fuse global and local features to characterize both local fine details and global structures within an image. The feature level fusion and decision level fusion are two popular types of fusion. In decision level fusion, voting may lead to a rough result in some cases. Thus, in this work, we have adopted feature level fusion. The feature level fusion creates curse of dimensionality, but in our case, size of feature vector is fixed and it is only 1632.

The main contributions made in this paper are as follows.

1. To the best of our knowledge, the local and global features are combined together for scene image classification. The complementary nature of these two types of features can effectively mitigate the shortcomings of local feature representation based methods and global feature representation based methods.

2. Confidence Co-occurrence Matrix (CCM) is introduced.

3. Classification accuracy is obtained for sport events dataset with the help of a feature descriptor. This descriptor is a combination of CCM, Histograms, LBP variations, and the Canny-Edge detector. The above mentioned combination of features is used first time for classification and measures are discussed in terms of precision, recall, sensitivity, specificity and F-Score.

\section{Proposed Approach}

This section is taken verbatrim from authors' own paper [15] to increase the readability.

$$
\begin{gathered}
\operatorname{LBP}(X, Y)=\sum_{i=0}^{n-1} s\left(n_{c}-n_{i}\right) 2^{i} \\
s(x)=1 \text { if } x>=0 \\
s(x)=0 \text { otherwise }
\end{gathered}
$$

The block diagram of Figure1 depicts both pre-processing and feature extraction processes altogether. In the beginning, we have retrieved R, G, and, B planes from the RGB image. The RGB image is converted into HSV image to extract $\mathrm{H}, \mathrm{S}$, and $\mathrm{V}$ planes. We have computed the Confidence Co-occurrence Matrix properties using various distance and direction measures for every plane of an RGB image. The Local Binary Pattern histogram with bin size 256 is computed for R, G, and B planes of an RGB image. We have used Canny Edge Detector algorithm with 0.2 as the threshold value so that the algorithm retains the most prominent edges. For all the planes of an RGB 


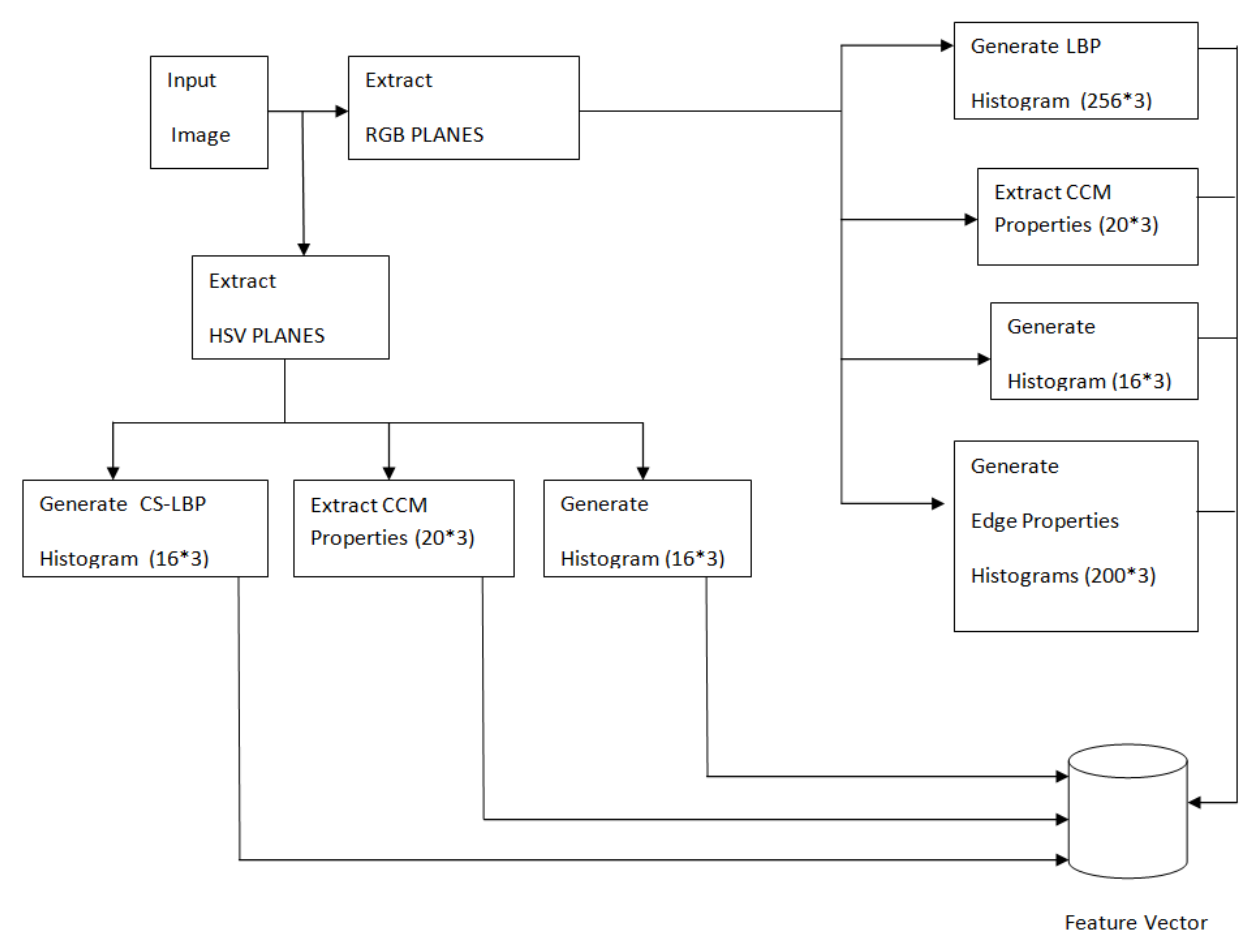

Figure 1. Proposed Approach

image, edge points have been extracted using Canny edge detector [10]. For every edge point, both the average and the variance have been calculated using $5 \times 5$ neighbourhood. We have computed the histogram of the mean values and a histogram of the variance values for every plane. We have extracted the sixteen bins Centre Symmetric Local Binary Pattern, the sixteen bins histogram of an image and properties of the Confidence Co-occurrence Matrix from an HSV image. The Local Binary Pattern captures texture information using the local neighbourhood. The LBP produces 256 distinct binary patterns. The histogram of the LBP has been separately calculated for the planes of the RGB image. The LBP histogram adds $768(256 \times * 3)$ additional features into the feature vector. variable 'nc' represents an intensity of a centre pixel of the 8-neighbourhood in the image. The variable 'ni' indicates the ith pixel of the neighbourhood. In the Centre Symmetric-LBP (CS-LBP), the centre-symmetric pairs of the pixel are compared. The CS-LBP generates 16 distinct binary patterns. In our proposed system, a histogram of CS-LBP is generated for all planes of an HSV image and generates $48(16 \times 3)$ more features[8][9]. A 'Confidence' shows how strong two or more entities are associated with each other in data mining. It is defined in terms of image processing as follows. Here, ' $\mathrm{B}$ ' is a gray level in an image of the second pixel which is at distance $\mathrm{D}$ and at angle $\theta$ from the first pixel having gray level 'A'. Confidence Co-occurrence Matrix is a modified version of Graylevel Co-occurrence Matrix.

$$
\operatorname{confidence}(A, B)=\frac{\operatorname{Count}(A, B)}{\operatorname{Count}(A)}
$$

Let's consider an example image of size 6x6 with 3 graylevels as given in Figure 2. It is clear from the Figure 2 that Confidence Co-occurrence matrix gives equal weight to both gray level combinations $(1,2)$ and $(3,2)$ which indicates equal importance. The Confidence Co-occurrence matrix also allocates more weight to gray level combination $(3,2)$ as compared to $(2,2)$ and $(2,3)$ gray level combinations while Generalized Co-occurrence matrix considers equally. It is also clear that gray level ' 3 ' appears with only gray level ' 2 ' while gray level '2' appears with all the gray levels thus gray level combination $(3,2)$ deserves more weight. 


\begin{tabular}{|l|l|l|l|l|l|}
\hline 1 & 2 & 3 & 2 & 1 & 2 \\
\hline 1 & 2 & 3 & 2 & 1 & 2 \\
\hline 1 & 2 & 3 & 2 & 1 & 2 \\
\hline 1 & 2 & 3 & 2 & 1 & 2 \\
\hline 1 & 2 & 2 & 2 & 1 & 2 \\
\hline 1 & 2 & 2 & 2 & 1 & 2 \\
\hline
\end{tabular}

(a)

\begin{tabular}{|l|l|l|l|}
\hline & 1 & 2 & 3 \\
\hline 1 & 0 & 12 & 0 \\
\hline 2 & 6 & 4 & 4 \\
\hline 3 & 0 & 4 & 0 \\
\hline
\end{tabular}

(b)

\begin{tabular}{|l|l|l|l|}
\hline & 1 & 2 & 3 \\
\hline 1 & 0 & 1 & 0 \\
\hline 2 & 0.3 & 0.20 & 0.20 \\
\hline 3 & 0 & 1 & 0 \\
\hline
\end{tabular}

(c)

Figure 2. (a) The Sample Image, (b) Generalized Co-occurrence Matrix with degree '0' and pixel distance 1, and (c) Confidence Co-occurrence Matrix.

Table 1. Confidence Co-occurrence Matrices constructed

\begin{tabular}{ccc}
\hline Number of Gray Levels & Distance & Direction \\
\hline 128 & 3 & Horizontal \\
\hline 128 & 9 & Horizontal \\
\hline 128 & 15 & Horizontal \\
\hline 128 & 64 & Horizontal \\
\hline 128 & 64 & Vertical \\
\hline
\end{tabular}

Contrast:

$$
\sum_{i, j}|i-j|^{2} C C M(i, j)
$$

Correlation:

$$
\sum_{i, j} \frac{\left(i-\mu_{i}\right)\left(j-\mu_{j}\right) C C M(i, j)}{\sigma_{i} \sigma_{j}}
$$

Energy:

$$
\sum_{i, j} C C M(i, j)^{2}
$$

Homogeneity:

$$
\sum_{i, j} \frac{C C M(i, j)}{1+|i-j|}
$$

The Confidence Co-occurrence Matrix is useful to extract texture of the image. Confidence Co-occurrence Matrix properties such as contrast, correlation, energy and homogeneity, with four different distances and two directions, are computed as described in Table 1 . This generates $120(20 \times 6)$ additional features for each plane of HSV and RGB image.

$\operatorname{CCM}(\mathrm{i}, \mathrm{j})$ represents an intensity at position $(\mathrm{i}, \mathrm{j}),{ }^{\prime} \mu$ ' denotes mean and ' $\sigma$ ' indicates the standard deviation in the above equations. An algorithm for calculation of Confidence co-occurrence Matrix has time complexity of $\theta(\mathrm{MN})$ 
Table 2. Sport Events Dataset

\begin{tabular}{ccc}
\hline No & Sport Events & Number of Images \\
\hline 1 & Rowing & 250 \\
\hline 2 & Badminton & 200 \\
\hline 3 & Polo & 182 \\
\hline 4 & Bocce & 137 \\
\hline 5 & Snowboarding & 190 \\
\hline 6 & Croquet & 236 \\
\hline 7 & Sailing & 190 \\
\hline 8 & Rock Climbing & 194 \\
\hline
\end{tabular}

is described here. Image I with size $\mathrm{M} \times \mathrm{N}$

$\mathrm{L}$ is number of gray levels

$\Delta \mathrm{x}$ is a displacement in $\mathrm{x}$-direction

$\Delta \mathrm{y}$ is a displacement in $\mathrm{y}$-direction

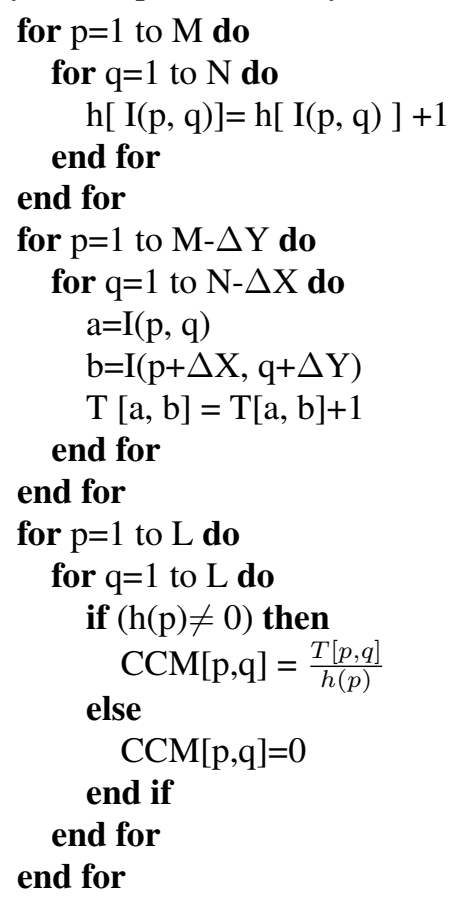

We have extracted the sixteen bins Centre Symmetric Local Binary Pattern, the sixteen bins histogram of an image and properties of the Confidence Co-occurrence Matrix from an HSV image.

The Local Binary Pattern captures texture information using the local neighbourhood. The LBP produces 256 distinct binary patterns. The histogram of the LBP has been separately calculated for the planes of the RGB image. The LBP histogram adds $768(256 \times * 3)$ additional features into the feature vector.

\section{Sport Events Dataset}

The dataset contains 8 sports event classes: rowing, badminton, polo, bocce, snowboarding, croquet, sailing, and rock climbing. This dataset is highly challenging. Difficulties associated with the dataset are listed below. The sample images are shown in Figure 3. Number of images for each class is tabulated in Table 2. 
1. The background of each image is highly cluttered and diverse;

2. Object classes are diverse;

3. Within the same category, sizes of instances from the same object are very different;

4. The pose of the object can be very different in each image;

5. Number of instances of the same object category change diversely within the same event category

6. Some of the foreground objects are too small to be detected.

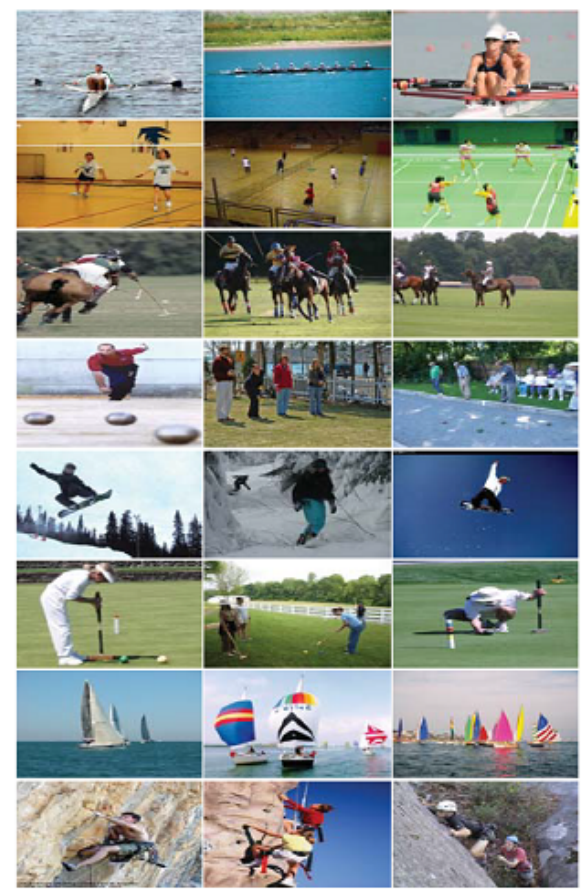

Figure 3. Sample images of Sport Dataset

\section{Flavia Dataset}

The Flavia Dataset contains 1907 images of leaves from 32 plant species on white background, 50 to 77 images per class. More than 30 research papers focus on classification of Flavia Leaf Dataset. The sample images are shown in Figure 4.

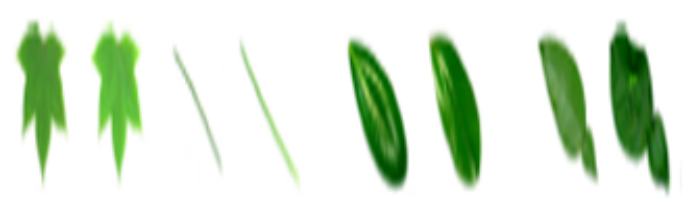

Figure 4. Sample images of Flavia Dataset 


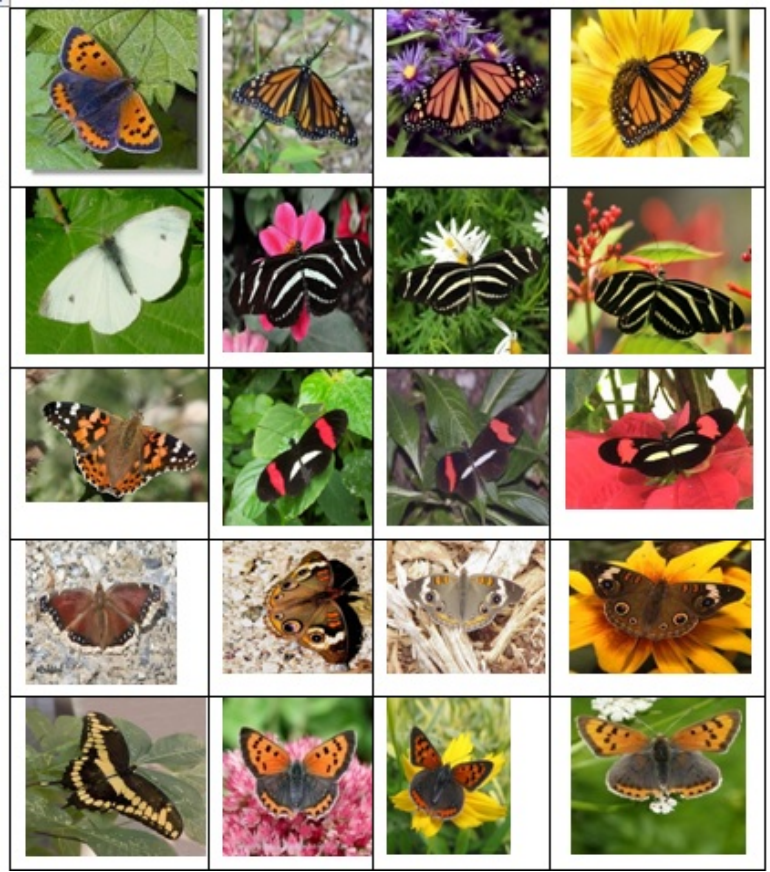

Figure 5. Sample images of Butterfly Dataset

Table 3. Leeds Butterfly Dataset

\begin{tabular}{cc}
\hline Leeds Butterfly Species & No. of samples \\
\hline Danaus plexippus & 82 \\
\hline Heliconius charitonius & 93 \\
\hline Heliconius erato & 61 \\
\hline Junonia coenia & 90 \\
\hline Lycaena phlaeas & 88 \\
\hline Nymphalis antiopa & 100 \\
\hline Nymphalis antiopa & 89 \\
\hline Pieris rapae & 55 \\
\hline Pieris rapae & 90 \\
\hline Vanessa cardui & 84
\end{tabular}

\section{Leeds Butterfly Dataset}

This dataset contains images and textual descriptions for ten categories (species) of butterflies. The image dataset comprises 832 images in total, with the distribution ranging from 55 to 100 images per category. Images were collected from Google Images by querying with the scientific (Latin) name of the species, for example "Danaus plexippus", and manually filtered for those depicting the butterfly of interest. The sample images are shown in Figure 5. Number of images for each class is tabulated in Table 3. 


\section{Birds Dataset}

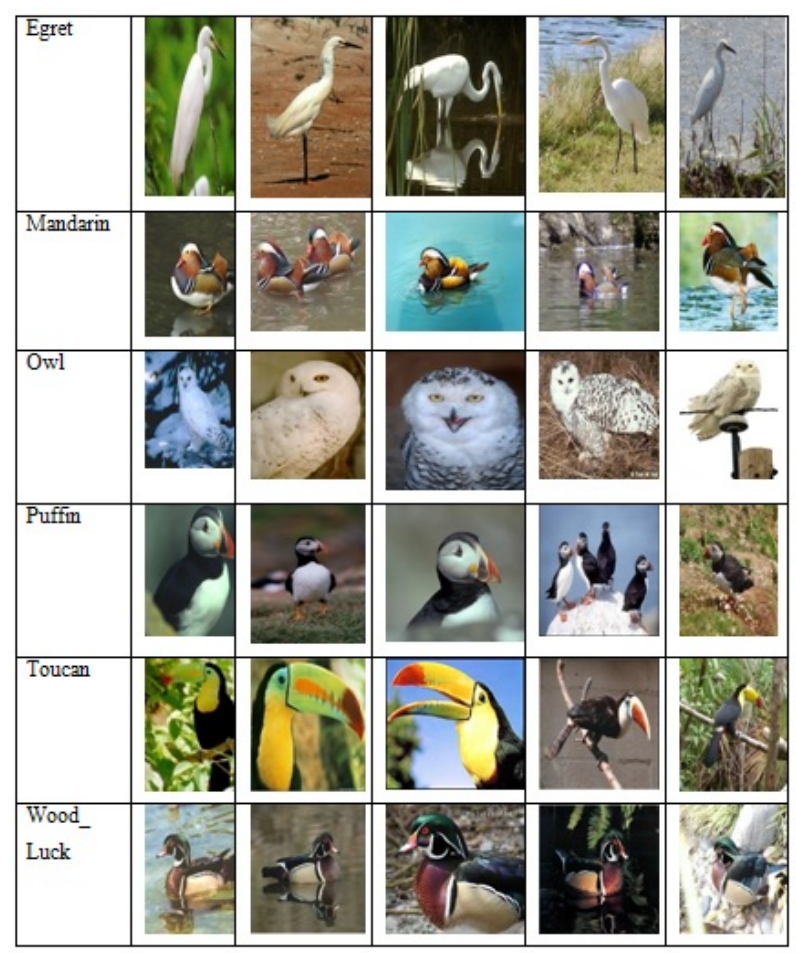

Figure 6. Sample images of Birds Dataset

This database contains 600 images (100 samples for each type of bird) of six different classes of birds(http://www-cvr.ai.uiuc.edu/ponce_grp/data/). The images are color JPEG, of variable resolution. Figure 6 shows five different samples of each bird class. Background in the image is not uniform. An image may contain 'Front View', 'Side View', 'Far View' or 'Near view' of the bird. Each image contains only one occurrence of the bird. Pose variation is also clearly visible. In a picture, an Egret bird may be in a standing position or it may be picking a food from the pond. A bird may be partly surrounded by tree leaves or may be occluded by any other objects.

\section{Experimental results}

Here, we have adopted classification accuracy calculated by a linear SVM classifier both on the training set as well as on the testing set. We have adopted 'holdout' method to partition the dataset into the training set and the testing set [12]. We have evaluated our proposed method using 64-bit MATLAB 2013a, 8GB of RAM running on the Windows 8.1 OS with i7 5th generation processor. The performance of the system is evaluated based on Precision, Recall, Accuracy and F-Score [11]. The results for the sport event dataset is tabulated in the Table4. The Table 5 shows accuracy achieved by various algorithms for the sport events dataset. Table 6 represents precision, recall, accuracy and F-score for the leeds butterfly dataset. Table7 and Table8 represent accuracy for the birds and the flavia dataset respectively. The Receiver Operating Characteristic Curves for the sport events dataset is shown in the Figure 7 while for the Leeds butterfly dataset is depicted in the Figure 8. 


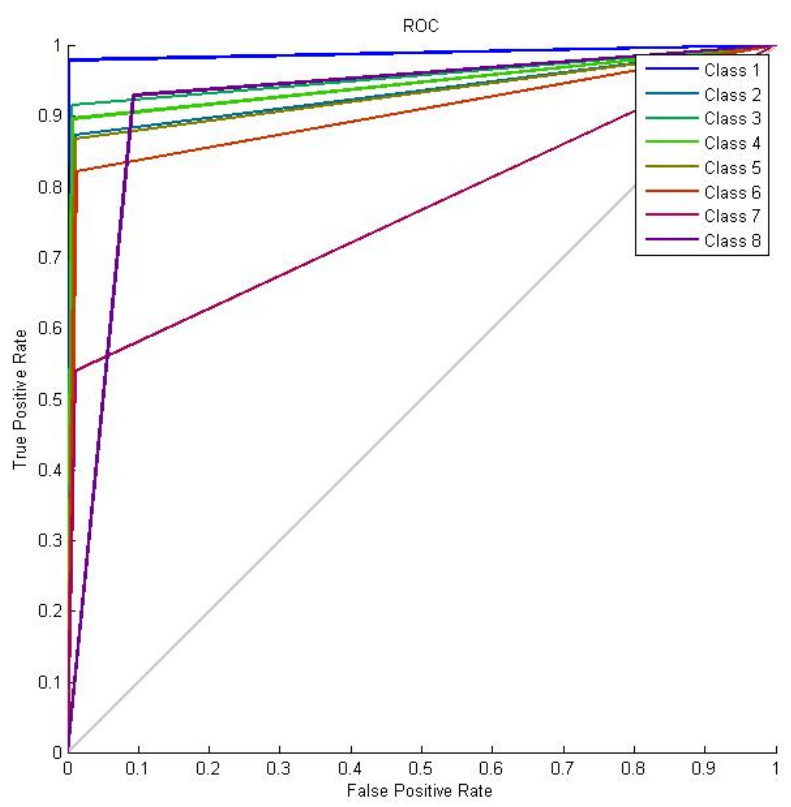

Figure 7. RoC Curve for Sport Events Dataset with $90 \%$ data in training \& $10 \%$ data in testing

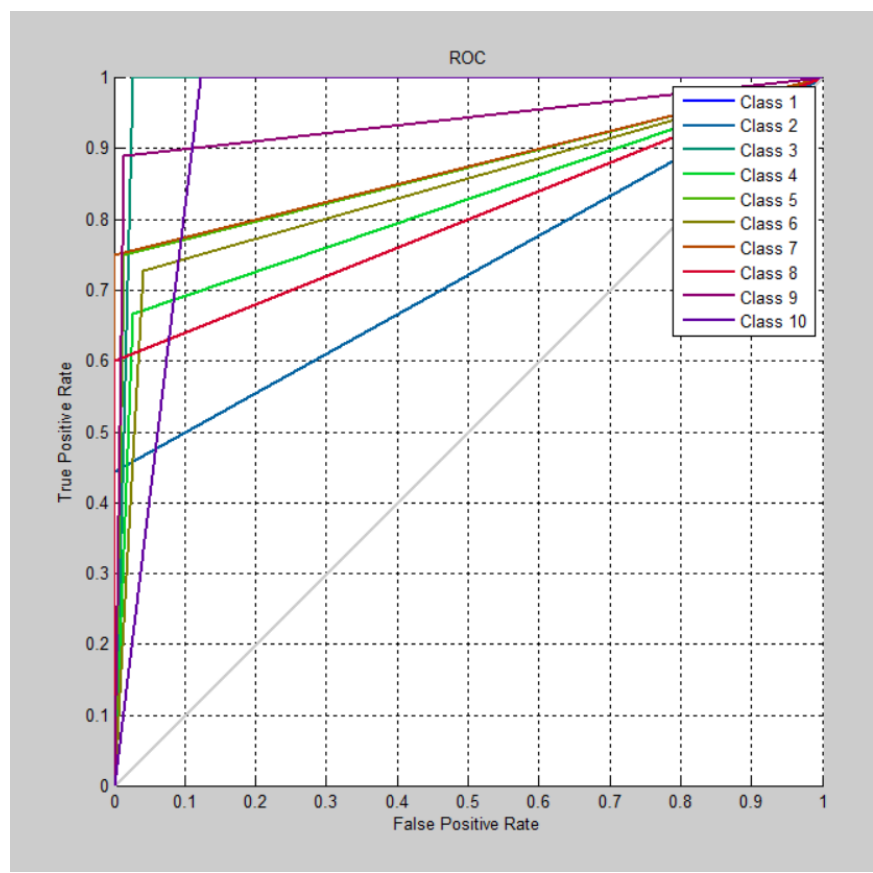

Figure 8. RoC Curve for Leeds Butterfly Dataset with 70\% data in training \& 30\% data in ttsting Data 
Table 4. Precision, Recall, Accuracy and F-score (Sport Events Dataset)

\begin{tabular}{ccccc}
\hline Sports Event & Precision & Recall & Accuracy & F-score \\
\hline Rowing & 0.99 & 0.96 & 0.99 & 0.98 \\
\hline Badminton & 0.95 & 0.83 & 0.59 & 0.69 \\
\hline Polo & 0.93 & 0.81 & 0.69 & 0.75 \\
\hline Bocce & 0.97 & 0.92 & 0.85 & 0.89 \\
\hline Snowboarding & 0.97 & 0.9 & 0.88 & 0.9 \\
\hline Croquet & 0.96 & 0.93 & 0.83 & 0.88 \\
\hline Sailing & 0.96 & 0.89 & 0.81 & 0.85 \\
\hline Rock climbing & 0.88 & 0.51 & 0.86 & 0.64 \\
\hline Average & $\mathbf{0 . 9 6}$ & $\mathbf{0 . 8 5}$ & $\mathbf{0 . 8 1}$ & $\mathbf{0 . 8 2}$ \\
\hline
\end{tabular}

Table 5. Accuracy Comparison(Sport Events Dataset)

\begin{tabular}{cc}
\hline Algorithms & Accuracy \\
\hline Full Integrative model [1] & 73.4 \\
\hline OB [2] & 76.3 \\
\hline SIFT+SC [3] & 82.7 \\
\hline HMP [4] & 85.7 \\
\hline MS-CLBP+KML [6] & 73.2 \\
\hline LLC+SVM [14] & 83.51 \\
\hline MS-CLBP+KCRC [14] & 84.1 \\
\hline LGF [14] & 88.52 \\
\hline Proposed (CCM+) & 96.1 \\
\hline
\end{tabular}


Table 6. Precision, Recall, Accuracy and F-score

\begin{tabular}{ccccc}
\hline Leeds Butterfly Species & Accuracy & Precision & Recall & F-score \\
\hline Danaus plexippus & 96.38 & 85.71 & 75 & 80 \\
\hline Heliconius charitonius & 93.97 & 70 & 77.78 & 73.68 \\
\hline Heliconius erato & 98.79 & 85.71 & 100 & 92.31 \\
\hline Junonia coenia & 92.77 & 75 & 60 & 66.67 \\
\hline Lycaena phlaeas & 93.97 & 71.43 & 62.5 & 66.67 \\
\hline Nymphalis antiopa & 97.59 & 90.91 & 90.91 & 90.91 \\
\hline Nymphalis antiopa & 96.38 & 85.71 & 75 & 80 \\
\hline Pieris rapae & 97.59 & 100 & 60 & 75 \\
\hline Pieris rapae & 93.97 & 83.33 & 55.56 & 66.67 \\
\hline Vanessa cardui & 87.95 & 47.06 & 88.89 & 61.54 \\
\hline Total & $\mathbf{9 4 . 9 4}$ & $\mathbf{7 9 . 4 9}$ & $\mathbf{7 4 . 5 6}$ & $\mathbf{7 5 . 3 4}$ \\
\hline
\end{tabular}

Table 7. Precision, Recall, Accuracy and F-score

\begin{tabular}{ccccc}
\hline Birds Dataset & Accuracy & Precision & Recall & F-Score \\
\hline Egret & 0.97 & 1 & 0.8 & 0.89 \\
\hline Mandarin & 0.95 & 0.89 & 0.8 & 0.84 \\
\hline Owl & 0.92 & 0.86 & 0.6 & 0.71 \\
\hline Puffin & 0.97 & 1 & 0.8 & 0.89 \\
\hline Toucan & 0.98 & 1 & 0.9 & 0.95 \\
\hline Wood_Luck & 0.91 & 0.53 & 1 & 0.69 \\
\hline Average & $\mathbf{0 . 9 5}$ & $\mathbf{0 . 8 8}$ & $\mathbf{0 . 8 2}$ & $\mathbf{0 . 8 3}$ \\
\hline
\end{tabular}


Table 8. Precision, Recall, Accuracy and F-score

\begin{tabular}{|c|c|c|c|c|}
\hline Flavia Dataset & Accuracy & Precision & Recall & F-score \\
\hline Pubescent Bamboo & 1 & 1 & 1 & 1 \\
\hline Chinese horse chestnut & 0.99 & 0.98 & 0.96 & 0.97 \\
\hline Anhui Barberry & 0.99 & 1 & 0.98 & 0.99 \\
\hline Chinese redbud & 1 & 1 & 1 & 1 \\
\hline Ture indigo & 0.99 & 1 & 0.98 & 0.99 \\
\hline Japanese maple & 1 & 1 & 1 & 1 \\
\hline Nanmu & 1 & 1 & 1 & 1 \\
\hline Castor aralia & 0.99 & 1 & 0.96 & 0.98 \\
\hline Chinese cinnamon & 0.99 & 1 & 0.96 & 0.98 \\
\hline Goldenrain tree & 1 & 1 & 1 & 1 \\
\hline Big-fruited holly & 0.99 & 0.98 & 0.98 & 0.98 \\
\hline Japanese cheesewood & 1 & 1 & 1 & 1 \\
\hline Winter sweet & 0.99 & 1 & 0.98 & 0.99 \\
\hline Camphortree & 0.99 & 1 & 0.92 & 0.96 \\
\hline Japan arrowood & 0.99 & 1 & 0.96 & 0.98 \\
\hline Sweet osmanthus & 1 & 1 & 1 & 1 \\
\hline Deodar & 1 & 1 & 1 & 1 \\
\hline Ginkgo,maidenhair tree & 0.99 & 1 & 0.98 & 0.99 \\
\hline Crape myrtle & 0.99 & 1 & 0.98 & 0.99 \\
\hline Oleander & 1 & 1 & 1 & 1 \\
\hline Yew plum pine & 1 & 1 & 1 & 1 \\
\hline Japansese flowering cherry & 0.99 & 1 & 0.9 & 0.95 \\
\hline Glossy privet & 0.99 & 1 & 0.98 & 0.99 \\
\hline Chinese toon & 0.99 & 1 & 0.98 & 0.99 \\
\hline Peach & 0.99 & 0.96 & 0.9 & 0.93 \\
\hline Ford woodlotus & 1 & 1 & 1 & 1 \\
\hline Tirdent maple & 1 & 1 & 1 & 1 \\
\hline Beale's barberry & 0.99 & 1 & 0.98 & 0.99 \\
\hline Southern mangolia & 1 & 1 & 1 & 1 \\
\hline Canadian poplar & 1 & 1 & 1 & 1 \\
\hline Chinese tulip tree & 0.99 & 1 & 0.96 & 0.98 \\
\hline Tangerine & 0.98 & 0.63 & 0.98 & 0.77 \\
\hline Average & 99 & 98.5 & 97.8 & 98.2 \\
\hline
\end{tabular}




\section{Conclusion}

In this paper, we have proposed Confidence Co-occurrence Matrix (CCM) which is a modification to existing Generalized Co-occurrence Matrix approach. When CCM is combined with LBP, CS-LBP, Histogram and other feature descriptors gives very high classification accuracy. We validated the proposed feature extraction method on four different datasets and quantitatively showed the excellence of the proposed method over some state-of-the-art classification methods.

\section{REFERENCES}

1. Li, L.J. and Fei-Fei, L What, where and who? classifying events by scene and object recognition In Proc. of IEEE 11th International Conference on Computer Vision, Brazil, pp. 1-8, 2007

2. L. Li , H. Su , E. Xing and F.-F. Li Object bank: A high-level image representation for scene classification and semantic feature sparsification In Proc. of the Neural Information Processing Systems, Vancouver, Canada, 2008

3. J. Yang , K. Yu , Y. Gong and T. Huang Linear spatial pyramid matching using sparse coding for image classification In Proc. of the IEEE Computer Society Conference on Computer Vision and Pattern Recognition, Miami, Florida, USA, 2009

4. L. Bo, X. Ren and D. Fox, Hierarchical matching pursuit for image classification: Architecture and fast algorithms In Proc. of Advances in Neural Information Processing Systems, 2011.

5. Fei-Fei. L, A. Iyer, C. Koch, and P. Perona, What do we perceive in a glance of a real-world scene? Journal of Vision, Vol.7, No.1, pp:1-29, 2007.

6. Chen, B. Zhang, H. Su, W. Li, L, Land-use scene classification using multi-scale completed local binary patterns In Proc. of Signal Image Video Process, 2015.

7. Sheng, G., Yan, W., Xu, T., and Sun, H., High-resolution satellite scene classification using a sparse coding based multiple feature combination International Journal of Remote Sensing, Vol.33, No. 8, pp. 2395-2412, 2012.

8. Heikkilä M, Pietikäinen M, Schmid C Description of interest regions with center-symmetric local binary patterns In Proc. of Computer vision, graphics and image processing. Springer Berlin Heidelberg, pp. 58-69, 2006.

9. Meena K, Suruliandi A, Rose RR, Face recognition based on local derivative ternary pattern IETE Journal of Research. Vol.60, No. 1, pp. 20-32, 2014

10. Syam B., ShrinivasRao Y. An effective similarity measure via genetic algorithm for Content-Based Image Retrieval with extensive features International Journal of Signal and Imaging Systems Engineering, Vol. 5, No. 1, pp. 18-28, 2012.

11. Jiawei Han, Micheline Kamber, and Jian Pei, Data Mining Concepts and Techniques Elsevier, 2011.

12. S. Manimala and K. Hemchandran Image Retrieval-Based on Colour Histogram and performance Evaluation of similarity Measurement. Assam University Journal of science and Technology, Vol. 8, pp.94-104.

13. N.Serrano, A.Savakis, and J. Luo, Improved scene classification using efficient low-level features and semantic cues. Pattern Recognition, Vol. 37, pp. 1773-1784, 2004

14. J. Zou, W. Li, C. Chen, and Q. Du Scene Classification Using Local and Global Features with Collaborative Representation Fusion Information Sciences, vol. 348, pp. 209-226, June 2016.

15. M.S.Bhatt and T.P.Patalia, Content-based high resolution satellite image clasification, International Journal of Information Technology, Vol. 1, no. 1, pp. 108-126, 2019.

16. M.S.Bhatt and T.P.Patalia, Indian monuments classification using support vector machine, International Journal of Electrical and Computer Engineering, Vol. 7, no. 4, pp. 1952-1963, 2017.

17. M.S.Bhatt and T.P.Patalia, Genetic programming evolved spatial descriptor for Indian monuments classification, In Proc. IEEE International Conference on Computer Graphics, Vision and Information Security, 2015.

18. S.R.Dubey and A.S.Jala, Species and variety detection of fruit and vegetables from images, International Journal of Applied Pattern Recognition, Vol. 1, no. 1, pp. 108-126, 2013.

19. S.R.Dubey and A.S.Jala, Fruit diseases recognition using improved sum and difference histogram from images, International Journal of Applied Pattern Recognition, Vol. 1, no. 2, pp. 199-220, 2014.

20. S.R.Dubey and A.S.Jala, Fruit and Vegetable recognition by fusing colour and texture features of the image using machine learning, International Journal of Applied Pattern Recognition, Vol. 2, no. 2, pp. 160-181, 2015.

21. Ping Zhong and Zhiqiang Gong, A Hybrid DBN and CRF Model for Spectral-Spatial Classification of Hyperspectral Images Statistics, Optimization and Information Computing. Vol. 5, No.2, pp.75-98, 2017. 